\title{
Impact of hypertension on the accuracy of exercise stress myocardial perfusion imaging for the diagnosis of coronary artery disease
}

\author{
A Elhendy, R T van Domburg, F B Sozzi, D Poldermans, J J Bax, J R T C Roelandt
}

\begin{abstract}
Aim-To compare the accuracy of exercise stress myocardial perfusion single photon emission computed tomography (SPECT) imaging for the diagnosis of coronary artery disease in patients with and without hypertension.

Methods-A symptom limited bicycle exercise stress test in conjunction with $99 \mathrm{~m}$ technetium sestamibi or tetrofosmin SPECT imaging was performed in 332 patients (mean (SD) age, 57 (10) years; 257 men, 75 women) without previous myocardial infarction who underwent coronary angiography. Of these, $137(41 \%)$ had hypertension. Rest SPECT images were acquired 24 hours after the stress test. An abnormal scan was defined as one with reversible or fixed perfusion defects.

Results-In hypertensive patients, myocardial perfusion abnormalities were detected in 79 of 102 patients with significant coronary artery disease and in nine of 35 patients without. In normotensive patients, myocardial perfusion abnormalities were detected in 104 of 138 patients with significant coronary artery disease and in 16 of 57 patients without. There were no differences between normotensive and hypertensive patients in sensitivity (77\% (95\% confidence interval (CI) $69 \%$ to $86 \%$ ) v $75 \%$ (95\% CI $68 \%$ to $83 \%)$ ), specificity $(74 \%(95 \%$ CI $60 \%$ to $89 \%) v 72 \%$ (95\% CI $60 \%$ to $84 \%)$ ), and accuracy ( $77 \%$ (95\% CI $70 \%$ to $84 \%$ ) v $74 \%(95 \%$ CI $68 \%$ to $80 \%)$ ) of exercise SPECT for diagnosing coronary artery disease. The accuracy of SPECT was greater than electrocardiography, both in hypertensive patients $(p=0.005)$ and in normotensive patients $(\mathrm{p}=0.0001)$. For the detection of coronary artery disease in individual vessels, sensitivity was $58 \%(95 \%$ CI $51 \%$ to $65 \%$ ) v $57 \%$ (95\% CI $51 \%$ to $64 \%$ ), specificity was $86 \%(95 \%$ CI $82 \%$ to $90 \%$ ) v $85 \%$ (95\% CI $81 \%$ to $89 \%$ ), and accuracy was $74 \%$ (95\% CI $70 \%$ to $78 \%$ ) v $74 \%$ (95\% CI $70 \%$ to $78 \%$ ) in patients with and without hypertension (NS).
\end{abstract}

Conclusions-In the usual clinical setting, the value of exercise myocardial perfusion scintigraphy for diagnosing coronary artery disease is not degraded by the presence of hypertension. (Heart 2001;85:655-661)

Keywords: hypertension; coronary artery disease; exercise stress test; myocardial perfusion

Evaluation of the accuracy of current techniques employed in non-invasive diagnosis is essential for the proper management and risk stratification of patients with suspected coronary artery disease. Exercise myocardial perfusion scintigraphy is widely used for this purpose. ${ }^{1-3}$ Hypertension is one of the most common risk factors for coronary artery disease encountered in patients undergoing exercise stress testing. ${ }^{4}$ Although exercise myocardial perfusion scintigraphy has been shown to be more accurate than exercise electrocardiography for the diagnosis of coronary artery disease, ${ }^{5}$ myocardial perfusion abnormalities may occur in hypertensive patients without significant epicardial coronary artery disease. ${ }^{6-8}$ Such abnormalities may not necessarily represent a false positive diagnosis of myocardial ischaemia as they may represent the sequelae of microvascular disease, impaired vasodilator reserve, and increased myocardial oxygen demand in a certain subset of hypertensive patients. ${ }^{6-11}$ However, the findings may result in unnecessary cardiac catheterisation in the absence of significant stenosis of the epicardial coronary arteries.

Although the occurrence of myocardial perfusion abnormalities in hypertensive patients without epicardial coronary artery disease is well documented, the impact of this observation on the specificity and value of exercise stress myocardial perfusion scintigraphy for diagnosing coronary artery disease in a routine clinical setting is far from clear, and it is not known whether myocardial perfusion scintigraphy suffers particular limitations in hypertensive patients. Such information is important for physicians in deciding whether to refer hypertensive patients for this type of imaging. The aim of our study was to compare the diagnostic accuracy of exercise stress single photon emission computed tomography (SPECT) with $99 \mathrm{~m}$ technetium labelled agents for the diagnosis of coronary artery disease in patients with and without hypertension.

\section{Methods}

PATIENT SELECTION

The study population consisted of 332 patients without previous myocardial infarction referred for the diagnosis of coronary artery disease by exercise stress SPECT imaging in our laboratory, who underwent coronary angiography within three months of the exercise stress test. Exclusion criteria were a history or ECG diagnosis of previous myocardial infarction, heart failure, unstable angina, severe valvar heart disease, and left bundle 
branch block. Of these patients, 137 (42\%) had systemic arterial hypertension, defined as repeated blood pressure measurements exceeding 140/90 mm Hg on different occasions, or being on antihypertensive drug treatment for a known diagnosis of hypertension. The diagnosis was confirmed by medical reports from the referring physicians. The mean (SD) duration of hypertension was 7.9 (5.7) years. Left ventricular hypertrophy by ECG criteria was detected in 20 patients. The mean age of the entire patient population was 57 (10) years. There were 257 men and 75 women.

\section{EXERCISE STRESS TEST}

All patients underwent a symptom limited upright bicycle ergometry test with stepwise increments of $20 \mathrm{~W}$ each minute. Blood pressure was measured every two minutes by the cuff method. Three ECG leads were monitored continuously. A 12 lead ECG was recorded at rest and every minute until the end of the recovery phase. The level of the ST segment was calculated by averaging the signals using a computerised system (Cardiovet, CSG/ 12, Schiller, Baar, Switzerland). An ischaemic response was defined as $\geqslant 0.1 \mathrm{mV}$ horizontal or downsloping ST segment depression or $\geqslant 0.1 \mathrm{mV}$ ST segment elevation compared with baseline, measured $80 \mathrm{~ms}$ from the J point.

SPECT IMAGING

Approximately one minute before termination of the exercise stress test, an intravenous dose of $370 \mathrm{MBq}$ of $99 \mathrm{~m}$ technetium methoxy isobutyl isonitrile sestamibi (233 patients) or tetrofosmin (99 patients) was given. Stress images were acquired one hour after termination of the test. For resting studies, $370 \mathrm{MBq}$ of the same isotope was injected 24 hours after the stress study. For each study six oblique (short axis) slices from the apex to the base and three sagittal (vertical long axis) slices from the septum to the lateral wall were defined. Each of the six short axis slices was divided into eight equal segments. The interpretation of the scan was performed semiquantitatively by visual analysis assisted by circumferential profile analysis. Myocardial perfusion was assessed by measuring the area between the lower limit of normal values ( \pm 2 SD) and the actual circumferential profile of the patient on rest and stress images. Stress and rest tomographic views were reviewed side by side by an experienced observer who was unaware of the patients' clinical, exercise stress, or angiographic data. A reversible perfusion defect was defined as a perfusion defect on stress images that partially or completely resolved at rest in two or more contiguous segments or slices. A fixed perfusion defect was defined as a perfusion defect on stress images in two or more contiguous segments or slices which persisted on rest images. There was no significant difference between patients with and without hypertension with respect to the proportion of patients who received tetrofosmin $(33 \% v$ $28 \%)$ or sestamibi $(67 \%$ v $72 \%)$.
CORONARY ANGIOGRAPHY

Coronary angiography was performed within three months of the exercise stress test. Lesions were quantified as previously described. ${ }^{12}$ Significant coronary artery disease was defined as a diameter stenosis of $\geqslant 50 \%$ in one or more major epicardial arteries. The anterior, apical, septal, and anteroseptal walls were assigned to the left anterior descending coronary artery. The posterior and lateral walls were assigned to the left circumflex coronary artery. The inferior and basal septal segments were assigned to the right coronary artery. The apical lateral segment was considered to be an overlap segment between the left anterior descending and the left circumflex arteries. The apical inferior segment was considered to be an overlap segment between the left anterior descending and the right coronary arteries. Overlapping segments were assigned to the regions with concomitant abnormalities.

ECHOCARDIOGRAPHIC ASSESSMENT OF LEFT

VENTRICULAR HYPERTROPHY

Echocardiography was performed in 117 hypertensive patients $(85 \%)$ within three months of the exercise stress test. Left ventricular mass was calculated using Troy's method, and measurements were made in accordance with the American Society of Echocardiography criteria $^{13}$ as follows: left ventricular mass $(\mathrm{g})=1.04$ $\left[(\text { IVS + LVID + PWT })^{3}-(\text { LVID })^{3}\right]$, where IVS $=$ interventricular septal thickness $(\mathrm{cm})$, LVID $=$ left ventricular internal dimension $(\mathrm{cm})$, and PWT = left ventricular posterior wall thickens (cm). The result was then corrected by the following equation ${ }^{14}$ to correlate with necropsy mass: left ventricular mass $(\mathrm{g})=0.8$ (LV mass) +0.6 . Left ventricular mass was indexed by body surface area using normal limits from the Framingham heart study. ${ }^{15}$ Left ventricular hypertrophy was defined as a left ventricular mass index of $>131 \mathrm{~g} / \mathrm{m}^{2}$ for men and $>100 \mathrm{~g} / \mathrm{m}^{2}$ for women.

\section{STATISTICAL ANALYSIS}

Unless specified, data are presented as mean (SD). The $\chi^{2}$ test was used to compare differences between proportions. Student's $t$ test was used for analysis of continuous data. Probability values of $\mathrm{p}<0.05$ were considered significant.

\section{Results}

\section{CLINICAL FEATURES}

Table 1 summarises the clinical features of patients with and without hypertension. There was no significant difference between the two groups for age, sex, symptoms, prevalence of hypercholesterolaemia and cigarette smoking, and treatment with $\beta$ blockers and nitrates. Hypertensive patients had a higher prevalence of diabetes mellitus and were more often on treatment with calcium channel blockers, diuretics, and angiotensin converting enzyme inhibitors than non-hypertensive patients. 
Table 1 Clinical features of patients with and without hypertension

\begin{tabular}{llll}
\hline & \multicolumn{2}{l}{ Hypertension } & \\
\cline { 2 - 3 } Clinical features & Yes $(n=137)$ & No $(n=195)$ & p Value \\
\hline Age (years) (mean (SD)) & $56(9)$ & $57(10)$ & 0.4 \\
Men & $101(74 \%)$ & $156(80 \%)$ & 0.2 \\
Reasons for referral & $58(42 \%)$ & $72(37 \%)$ & 0.3 \\
$\quad$ Typical angina & $44(32 \%)$ & $59(30 \%)$ & 0.7 \\
Atypical angina & $35(26 \%)$ & $64(33 \%)$ & 0.2 \\
Non-anginal chest pain & $19(14 \%)$ & $12(6 \%)$ & 0.02 \\
Diabetes mellitus & $30(22 \%)$ & $53(27 \%)$ & 0.3 \\
Hypercholesterolaemia & $27(20 \%)$ & $55(28 \%)$ & 0.07 \\
$\quad$ Cigarette smoking & $69(50 \%)$ & $92(47 \%)$ & 0.6 \\
Drug treatment & $99(72 \%)$ & $39(20 \%)$ & 0.0001 \\
$\quad \beta$ Blockers & $37(27 \%)$ & $49(25 \%)$ & 0.7 \\
Calcium channel blockers & $29(21 \%)$ & $3(2 \%)$ & 0.0001 \\
$\quad$ Nitrates & $22(16 \%)$ & $0(\%)$ & 0.0001 \\
ACE inhibitors & & \\
Diuretics & &
\end{tabular}

Values are $\mathrm{n}(\%)$ unless specified.

$\mathrm{ACE}$, angiotensin converting enzyme.

Table 2 Haemodynamic data of patients with and without hypertension

\begin{tabular}{llll}
\hline & \multicolumn{2}{l}{ Hypertension } & \\
\cline { 2 - 3 } Haemodynamic and stress test variables & Yes $(n=137)$ & \multirow{2}{*}{ No $(n=195)$} & p Value \\
\hline Heart rate at rest (beats/min) & $81(22)$ & $74(19)$ & 0.002 \\
Heart rate at peak stress (beats/min) & $137(23)$ & $138(23)$ & 0.8 \\
Systolic blood pressure at rest (mm Hg) & $147(20)$ & $130(19)$ & 0.0001 \\
Systolic blood pressure at peak stress (mm Hg) & $188(26)$ & $180(24)$ & 0.02 \\
Diastolic blood pressure at rest (mm Hg) & $91(13)$ & $81(10)$ & 0.0001 \\
Diastolic blood pressure at peak stress (mm Hg) & $92(16)$ & $88(12)$ & 0.0001 \\
Rate-pressure product at rest & $11975(3280)$ & $9807(2910)$ & 0.0001 \\
Rate-pressure product at peak stress & $25763(6237)$ & $24850(6322)$ & 0.4 \\
》 85\% of maximum heart rate achieved (n (\%)) & $84(61 \%)$ & $113(58 \%)$ & 0.5 \\
Mean working capacity (W) & $139(40)$ & $143(39)$ & 0.1 \\
ST segment depression during the test (n (\%)) & $76(55 \%)$ & $91(47 \%)$ & 0.09 \\
Angina during the test (n (\%)) & $37(27 \%)$ & $49(25 \%)$ & 0.7 \\
\hline
\end{tabular}

Values are mean (SD) unless specified.

Table 3 Coronary angiographic data of patients with and without hypertension

\begin{tabular}{llll}
\hline & \multicolumn{2}{l}{ Hypertension } & \\
\cline { 2 - 3 } Coronary angiographic variables & Yes $(n=137)$ & No $(n=195)$ & p Value \\
\hline Significant CAD & $102(74 \%)$ & $138(71 \%)$ & 0.5 \\
Single vessel CAD & $54(39 \%)$ & $76(39 \%)$ & 0.9 \\
Two vessel CAD & $22(16 \%)$ & $31(16 \%)$ & 0.9 \\
Three vessel CAD & $26(19 \%)$ & $31(16 \%)$ & 0.5 \\
Normal coronary arteries or < 50\% lesion & $35(26 \%)$ & $57(29 \%)$ & 0.5 \\
$\geqslant 70 \%$ stenosis in $\geqslant 1$ coronary artery & $66(48 \%)$ & $87(45 \%)$ & 0.5 \\
LAD stenosis & $65(47 \%)$ & $93(48 \%)$ & 0.9 \\
LCx stenosis & $51(37 \%)$ & $62(32 \%)$ & 0.3 \\
RCA stenosis & $60(44 \%)$ & $76(39 \%)$ & 0.4 \\
\hline
\end{tabular}

$\mathrm{CAD}$, coronary artery disease; LAD, left anterior descending coronary artery; LCx, left circumflex coronary artery; RCA, right coronary artery.

Table 4 Accuracy of exercise stress SPECT for the diagnosis of significant coronary artery stenosis in patients with and without hypertension

\begin{tabular}{|c|c|c|c|c|}
\hline & $\begin{array}{l}\text { Hypertension } \\
(n=137)\end{array}$ & Numbers & $\begin{array}{l}\text { No hypertension } \\
(n=195)\end{array}$ & Numbers \\
\hline \multicolumn{5}{|c|}{ Overall diagnosis of coronary artery disease } \\
\hline Sensitivity & 77 (69 to 86$)$ & $79 / 102$ & 75 (68 to 83 ) & $104 / 138$ \\
\hline Specificity & $74(60$ to 89$)$ & $26 / 35$ & $72(60$ to 84$)$ & $41 / 57$ \\
\hline Accuracy & $77(70$ to 84$)$ & 105137 & $74(68$ to 80$)$ & $145 / 195$ \\
\hline \multicolumn{5}{|l|}{ LAD stenosis } \\
\hline Sensitivity & $63(51$ to 75$)$ & $41 / 65$ & $62(53$ to 72$)$ & $58 / 93$ \\
\hline Specificity & $88(80$ to 95$)$ & $63 / 72$ & $84(77$ to 91$)$ & $86 / 102$ \\
\hline Accuracy & $76(69$ to 83$)$ & $104 / 137$ & $74(68$ to 80$)$ & $144 / 195$ \\
\hline \multicolumn{5}{|l|}{ LCx stenosis } \\
\hline Sensitivity & $59(45$ to 72$)$ & $30 / 51$ & 53 (41 to 66$)$ & $33 / 62$ \\
\hline Specificity & $83(75$ to 91$)$ & $71 / 86$ & 86 (80 to 92$)$ & $114 / 133$ \\
\hline Accuracy & $74(66$ to 81$)$ & $101 / 137$ & $75(69$ to 81$)$ & $147 / 195$ \\
\hline \multicolumn{5}{|l|}{ RCA stenosis } \\
\hline Sensitivity & $52(39$ to 64$)$ & $31 / 60$ & $54(43$ to 65$)$ & $41 / 76$ \\
\hline Specificity & $88(81$ to 95$)$ & $68 / 77$ & $85(78$ to 91$)$ & $101 / 119$ \\
\hline Accuracy & 72 (65 to 80$)$ & $99 / 137$ & 73 (67 to 79$)$ & $142 / 195$ \\
\hline \multicolumn{5}{|l|}{ All arteries } \\
\hline Sensitivity & $58(51$ to 65$)$ & $102 / 176$ & $57(51$ to 64$)$ & $132 / 231$ \\
\hline Specificity & $86(82$ to 90$)$ & $202 / 235$ & $85(81$ to 89$)$ & $301 / 354$ \\
\hline Accuracy & 74 (70 to 78$)$ & $304 / 411$ & $74(70$ to 78$)$ & $433 / 585$ \\
\hline
\end{tabular}

Values are percentages $(95 \% \mathrm{CI})$.
SYMPTOMS AND HAEMODYNAMIC RESPONSE

Table 2 summarises the symptoms and the haemodynamic and electrocardiographic responses in patients with and without hypertension. Resting heart rate, systolic and diastolic blood pressure, and rate-pressure product were significantly higher in patients with hypertension. Hypertensive patients achieved a higher peak systolic and diastolic blood pressure, although the increment in systolic blood pressure was more significant in patients without hypertension $(p<0.005)$. Peak heart rate, ratepressure product, and the proportion of patients who achieved the target heart rate or had exercise induced angina, were not significantly different between the two groups.

\section{ANGIOGRAPHIC FINDINGS}

Significant coronary artery disease was detected in 240 patients $(72 \%)$. Data on its extent and location in patients with and without hypertension are given in table 3. There was no significant difference in prevalence or distribution between the groups. No cardiac events or worsening of symptoms occurred in the interval between the exercise stress test and coronary angiography.

ACCURACY OF EXERCISE SPECT FOR THE DIAGNOSIS AND LOCALISATION OF CORONARY ARTERY DISEASE

Patients with hypertension

Myocardial perfusion abnormalities (reversible or fixed perfusion defects) were detected in 79 of 102 patients with significant coronary artery disease and in nine of 35 patients without coronary disease (sensitivity $77 \%$, (95\% confidence interval (CI) $69 \%$ to $86 \%$ ); specificity $74 \%$ (95\% CI $60 \%$ to $89 \%$ ); accuracy $77 \%$ (95\% CI $70 \%$ to $84 \%$ )) (table 4 ). In patients with single vessel disease, sensitivity was $72 \%$ $(39 / 54)$, with two vessel disease it was $82 \%$ $(18 / 22)$, and with three vessel disease it was $85 \%(22 / 26)$. Myocardial perfusion abnormalities in two vascular regions, suggestive of multivessel coronary artery disease, were detected in 25 of 48 patients with multivessel disease and in six of 89 patients without multivessel disease (sensitivity for detecting coronary stenosis in more than one vascular region $52 \%$ (95\% CI $38 \%$ to $66 \%$ ); specificity $93 \%$ (95\% CI $88 \%$ to $98 \%$ ); accuracy $79 \%$ (95\% CI $72 \%$ to $86 \%)$ ). In hypertensive patients without significant coronary artery disease, there was no difference between patients with and without perfusion abnormalities with regard to resting systolic blood pressure: 144 (21) v 146 (19) $\mathrm{mm} \mathrm{Hg}$ (NS).

\section{Patients without hypertension}

Myocardial perfusion abnormalities were detected in 104 of 138 patients with significant coronary artery disease and in 16 of 57 patients without coronary disease (sensitivity 75\% (95\% CI $68 \%$ to $83 \%$ ); specificity $72 \%$ (95\% CI $60 \%$ to $84 \%$ ); accuracy $74 \%$ (95\% CI $68 \%$ to $80 \%)$ ). In patients with single vessel disease, sensitivity was $67 \%(51 / 76)$, with two vessel disease it was $81 \%(25 / 31)$, and with three vessel disease it was $90 \%$ (28/31). Myocardial 


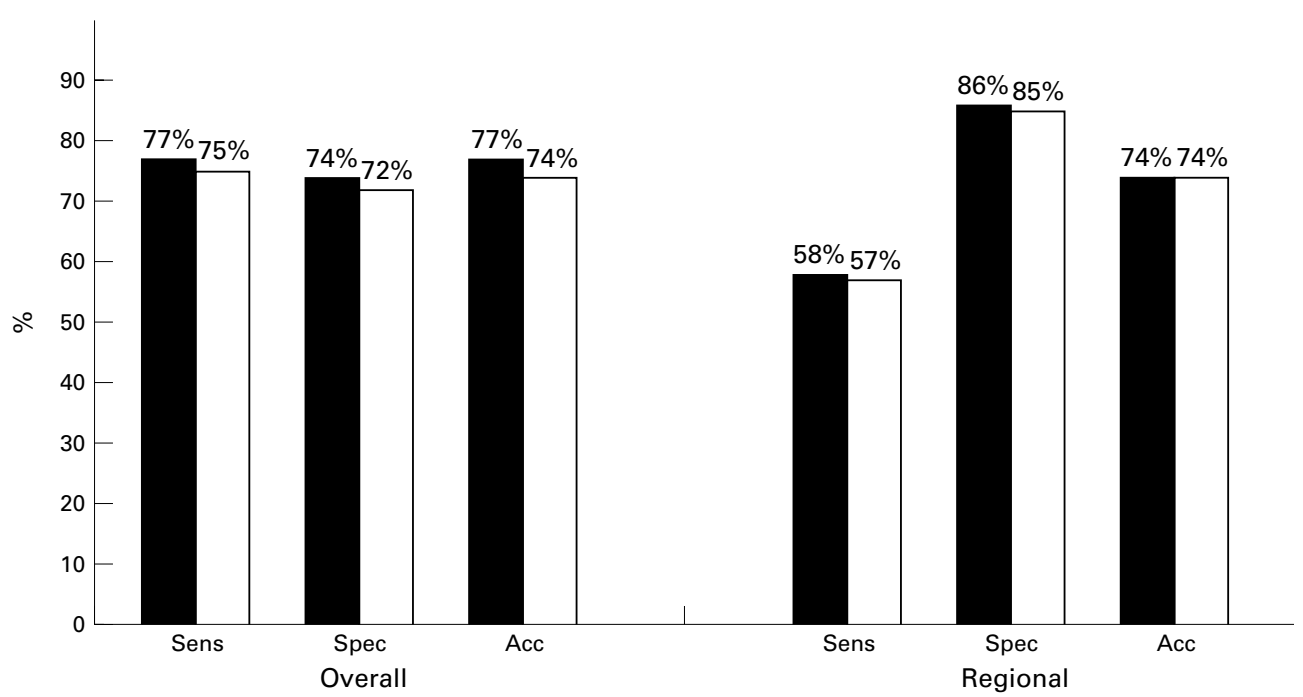

Figure 1 Sensitivity (Sens), specificity (Spec), and accuracy (Acc) of exercise stress SPECT for the overall and regional diagnosis of coronary artery disease in patients with hypertension (filled graph) and without hypertension (empty graph).

perfusion abnormalities in two vascular regions, suggestive of multivessel coronary artery disease, were detected in 32 of 62 patients with multivessel disease and in 13 of 133 patients without multivessel disease (sensitivity for detecting coronary stenosis in more than one vascular region $52 \%$ (95\% CI $39 \%$ to $64 \%$ ); specificity $90 \%$ (95\% CI $85 \%$ to $95 \%)$; accuracy $78 \%(95 \%$ CI $72 \%$ to $84 \%)$ ).

COMPARISON OF PATIENTS WITH AND WITHOUT HYPERTENSION

There was no significant difference between patients with and without hypertension with respect to sensitivity, specificity, and accuracy for the overall and regional diagnosis of coronary artery disease (table 4). Diagnostic accuracy did not differ among different vascular regions (fig 1). The accuracy of the diagnosis of multivessel coronary artery disease on the basis of perfusion abnormalities in more than one vascular region was not significantly different between the groups. In patients with a low to intermediate pretest probability of coronary artery disease, there was no significant difference between those with hypertension (75 patients) and those without hypertension (111 patients) in the sensitivity $(74 \%(35 / 47) v 72 \%$ $(49 / 68))$ or specificity $(75 \%(21 / 28)$ v $72 \%$ (31/43)) of SPECT imaging for diagnosing coronary artery disease. Similarly, in patients with a high pretest probability of coronary artery disease, there was no significant difference between those with hypertension (62 patients) and those without hypertension (84 patients) in the sensitivity $(80 \%(44 / 55) v 79 \%$ $(55 / 70))$ or specificity $(71 \% \quad(5 / 7)$ v $71 \%$ $(10 / 14))$ of SPECT imaging.

PATIENTS WITH FALSE NEGATIVE STUDIES

In patients with coronary artery disease, those with false negative results (57 patients) had a higher prevalence of single vessel coronary artery disease $(70 \% v 49 \%, \mathrm{p}<0.01)$ and a lower peak heart rate (123 (28) v 132 (24), $\mathrm{p}<0.05)$ than the 183 patients with a true positive test (abnormal perfusion).
IMPACT OF LEFT VENTRICULAR HYPERTROPHY Echocardiographic criteria of left ventricular hypertrophy were fulfilled in 57 of the 117 hypertensive patients (49\%) who underwent echocardiographic imaging. Significant coronary artery disease was detected in 41 of the 57 patients with left ventricular hypertrophy and in 43 of the 60 patients without. In patients with left ventricular hypertrophy, myocardial perfusion abnormalities were detected in 31 of the 41 patients with coronary artery disease and in four of the 16 patients without coronary disease. In patients without left ventricular hypertrophy, myocardial perfusion abnormalities were detected in 31 of the 43 patients with coronary disease and in four of the 17 patients without coronary disease. No significant difference was found between patients with and without left ventricular hypertrophy in the sensitivity $(76 \%$ (95\% CI $62 \%$ to $89 \%$ ) v $72 \%$ (95\% CI $59 \%$ to $85 \%)$ ), specificity $(75 \%(95 \%$ CI $54 \%$ to $96 \%)$ v $76 \%$ (95\% CI $62 \%$ to $77 \%)$ ), and accuracy (75\% (95\% CI $64 \%$ to $87 \%)$ v $73 \%(95 \%$ CI $62 \%$ to $85 \%)$ ) of exercise SPECT for diagnosing coronary artery disease (NS for all).

COMPARISON OF MYOCARDIAL PERFUSION SCINTIGRAPHY AND ELECTROCARDIOGRAPHY In hypertensive patients, ischaemic ECG changes were detected in 59 of 102 patients with significant coronary artery disease and in 17 of 35 patients without coronary disease (sensitivity $58 \% \quad(95 \%$ CI $48 \%$ to $67 \%)$, $\mathrm{p}=0.03 v$ SPECT; specificity 51\% (95\% CI $35 \%$ to $68 \%), p=0.04 v$ SPECT; accuracy $56 \%$ (95\% CI $48 \%$ to $65 \%), p=0.0005 v$ SPECT). In patients without hypertension, ischaemic ECG changes occurred in 73 of 138 patients with significant coronary artery disease and in 18 of 57 patients without coronary disease (sensitivity 53\% (95\% CI $45 \%$ to $61 \%), p=0.0001 v$ SPECT; specificity $68 \%$ (95\% CI $56 \%$ to $80 \%$ ); accuracy $57 \%$ (95\% CI $50 \%$ to $64 \%), p=0.0001 v$ SPECT). There was a trend towards a higher specificity of ECG changes in normotensive than in hypertensive 
patients, but the difference did not reach statistical significance $(68 \%$ v $51 \%, \mathrm{p}=0.1)$.

\section{Discussion}

Coronary artery disease is a major cause of morbidity and mortality in patients with systemic arterial hypertension. ${ }^{4}$ The accuracy of non-invasive methods employed in diagnosis and functional evaluation has a strong impact on the management and risk stratification of patients with known or suspected coronary artery disease. ${ }^{16}$ Exercise electrocardiography is widely used in making a diagnosis of coronary artery disease, but the relatively low specificity of the ECG changes in patients with hypertension limits its value under those circumstances. ${ }^{16}$ Exercise myocardial perfusion scintigraphy has been shown to provide a higher degree of diagnostic accuracy than electrocardiography in hypertensive patients. ${ }^{17}$ However, myocardial perfusion abnormalities are reported to occur in hypertensive patients without significant epicardial coronary arterial stenosis. ${ }^{6-8}$ Although myocardial perfusion abnormalities are well documented in hypertensive patients without significant coronary artery disease, previous studies have involved highly selected populations. It is not known whether myocardial perfusion scintigraphy suffers from any particular limitation for the diagnosis of coronary artery disease in hypertensive patients in a routine clinical setting.

PRESENT STUDY

To our knowledge, this is the first study evaluating the impact of systemic arterial hypertension on the accuracy of exercise stress myocardial perfusion imaging with $99 \mathrm{~m}$ technetium labelled agents for diagnosing and localising coronary artery disease. We found that exercise SPECT imaging provided a relatively high sensitivity and specificity for the overall diagnosis of coronary artery disease. The diagnosis of disease in individual coronary artery territories was accomplished with moderate sensitivity and high specificity, and did not differ among the three vascular regions. This confirms the value of SPECT imaging in the correct diagnosis of regional myocardial perfusion abnormalities.

There was no significant difference between patients with and without hypertension in the sensitivity, specificity, or accuracy of exercise SPECT for overall and regional diagnosis of coronary artery disease. Similarly, the accuracy of diagnosing multivessel coronary artery disease on the basis of perfusion abnormalities in more than one vascular region was not significantly different between the two groups. SPECT imaging provided a higher sensitivity and accuracy than electrocardiography irrespective of whether there was hypertension. In hypertensive patients, the specificity of SPECT was higher than that of electrocardiography. Among hypertensive patients, the presence of left ventricular hypertrophy did not affect the sensitivity or specificity of the diagnosis of coronary artery disease.

These data show that in hypertensive patients without previous myocardial infarction referred for the diagnosis of coronary artery disease, exercise SPECT myocardial perfusion imaging is a useful method for the overall and regional diagnosis of coronary artery disease, with comparable sensitivity and specificity to those achieved in normotensive patients. The similar specificity in the three vascular regions indicates that false positive results do not tend to involve a particular myocardial segment. Patients with coronary artery disease but normal perfusion were more likely to have single vessel disease and they had a lower exercise heart rate compared with patients who had perfusion abnormalities.

Seventeen of the 110 patients (15\%) with multivessel coronary artery disease had no perfusion abnormalities. This may be a reflection of the lower exercise heart rate achieved or of the effect of drugs on the induction of ischaemia. Alternatively, flow heterogeneity may not have been evident because of a diffuse pattern of hypoperfusion. Perhaps a gated study might have detected functional abnormalities in patients with a diffuse pattern of hypoperfusion.

COMPARISON WITH PREVIOUS STUDIES

Abnormalities of myocardial perfusion have been reported in hypertensive patients with or without left ventricular hypertrophy in the absence of significant epicardial coronary artery disease. ${ }^{6-8}$ The occurrence of myocardial ischaemia in these patients has been attributed to microvascular coronary artery disease, impaired vasodilator reserve, increased myocardial oxygen demand because of left ventricular hypertrophy, increased afterload, and diastolic dysfunction. ${ }^{10}$ DePuey and colleagues reported that fixed perfusion defects in the lateral wall often occur in hypertensive patients with left ventricular hypertrophy accompanying end stage renal disease. ${ }^{8}$ In contrast, Cecil and colleagues found no thallium perfusion abnormalities in 16 hypertensive patients with left ventricular hypertrophy but without renal disease. ${ }^{18}$ Schulman and associates compared the results of exercise thallium scintigraphy in patients with and without hypertension. ${ }^{19}$ They concluded that hypertension affects the results of thallium-201 exercise stress testing in patients with low but not with medium to high likelihood of coronary artery disease. In contrast, Grogan and colleagues reported that in patients with a low likelihood of coronary artery disease, the prevalence and extent of exercise thallium perfusion abnormalities were similar in normotensive and hypertensive patients. ${ }^{20}$

These previous studies compared the prevalence of myocardial perfusion abnormalities in patients with and without hypertension, and no correlations with coronary angiography were reported. Chin and colleagues studied 30 asymptomatic hypertensive patients who had a positive exercise ECG or thallium-201 scintigram and underwent coronary angiography. ${ }^{21}$ These investigators concluded that thallium201 scintigraphy can accurately diagnose coronary artery disease in most of the patients with asymptomatic essential hypertension, and 
that most asymptomatic hypertensive patients with physiological evidence of myocardial ischaemia have associated coronary artery disease. Fragasso and associates reported that in 101 patients with hypertension, chest pain, and a positive exercise ECG, stress sestamibi imaging had a lower specificity than dobutamine or dipyridamole echocardiography for diagnosing coronary artery disease..$^{22}$ However, the population in that study was highly selected as it included only patients with a positive exercise ECG; these represent only a proportion of hypertensive patients with suspected coronary artery disease.

Zouridakis and colleagues studied normotensive patients and hypertensive patients with angina, a positive exercise ECG, and normal coronary arteries. ${ }^{23}$ Dobutamine stress echocardiography was normal in all patients, whereas eight normotensive patients and 10 hypertensive patients had perfusion abnormalities. In contrast to their findings, we have previously reported that dobutamine stress echocardiography and simultaneous sestamibi SPECT had similar accuracy in diagnosing coronary artery disease in 84 hypertensive patients unable to perform an exercise stress test. ${ }^{24}$ The prevalence of myocardial perfusion abnormalities in patients with and without hypertension undergoing dobutamine stress myocardial perfusion imaging was found to be similar, regardless of the pretest probability of coronary artery disease. ${ }^{25}$

Our finding that the specificity of myocardial perfusion imaging was not reduced in patients with hypertension can be explained in various ways. Although myocardial perfusion abnormalities have been reported in hypertensive patients without significant epicardial coronary artery disease, the exact prevalence of these findings in hypertensive patients with suspected coronary artery disease is not known, as previous studies evaluated a highly selected population. It is possible that the prevalence of these abnormalities is not high enough to account for a difference in the specificity of myocardial perfusion imaging between patients with and without hypertension. On the other hand, perfusion abnormalities resulting from microvascular ischaemia have also been reported in patients without hypertension. ${ }^{26}$ Increased awareness of the cardiovascular complications of hypertension and advances in medical treatment, including the introduction of safe and effective antihypertensive drugs, ${ }^{4}$ are expected to attenuate the pathophysiological effects of hypertension on the myocardium, thereby reducing the severity of structural changes occurring in the absence of epicardial coronary artery disease. ${ }^{27}$ Finally, the impairment of coronary flow reserve in hypertensive patients without obstructive coronary artery disease is a diffuse process. Therefore mild diffuse attenuation of perfusion may result in apparently normal perfusion in patients without large vessel coronary artery disease. It is also possible that in most hypertensive patients without significant coronary artery disease the reduction in coronary flow reserve is not severe enough to account for a significant impairment of the radioactive isotope uptake during exercise, as maximum tracer uptake can occur at a submaximal level of coronary vasodilatation.

PREVIOUS STUDIES OF EXERCISE SPECT

The reported sensitivity of exercise sestamibi SPECT in previous studies ranges between $82 \%$ and $93 \%$. Specificity ranges between 36\% and $71 \%{ }^{27-31}$ Most of these studies were performed in a heterogeneous population with or without previous myocardial infarction. Among the studies which recruited more than 100 patients, Van Train and colleagues reported a sensitivity of $90 \%$ and specificity of $36 \%$ in 124 patients (19\% had a previous myocardial infarct) who underwent exercise sestamibi SPECT and coronary angiography. ${ }^{29}$ Solot and associates reported a sensitivity of $97 \%$ and specificity of $71 \%$ for exercise sestamibi SPECT in 128 patients. ${ }^{31}$ Previous studies of exercise tetrofosmin imaging reported a sensitivity ranging between $58 \%$ and $95 \%$ and a specificity ranging between 54\% and $95 \%$ for the diagnosis of coronary artery disease. ${ }^{32-34}$

\section{LIMITATIONS}

The effect of drug treatments on the results of our study should be taken into account. The administration of antihypertensive drugs may attenuate the pathophysiological mechanisms involved in the genesis of myocardial ischaemia in the absence of coronary artery disease. ${ }^{27}$ However, in clinical practice, patients with hypertension usually receive the appropriate treatment for hypertension before being evaluated for coronary artery disease, and therefore the study patients represent the hypertensive population with suspected coronary artery disease encountered in clinical practice. In such patients, non-invasive evaluation of coronary artery disease is warranted. Another aspect of drug treatment is the possible attenuation of myocardial ischaemia, with subsequent reduction in the sensitivity of exercise SPECT for diagnosing coronary artery disease. However, an underestimation of the true sensitivity of the test in our study does not detract from our conclusion about the clinical value of exercise myocardial perfusion scintigraphy for diagnosing coronary artery disease in hypertensive patients.

Although we used two different tracers in the study, previous studies have shown comparable performance of sestamibi and tetrofosmin in diagnosing coronary artery disease. ${ }^{35} 36$

Referral bias for coronary angiography may have influenced our results. However, this does not affect the main conclusion of the study, as selective referral of patients with a positive scan for coronary angiography is expected to reduce specificity, and the study already showed a similar and acceptable specificity in patients with and without hypertension.

Finally, there are limitations in the use of coronary angiography as a gold standard for the presence of functionally significant coronary artery disease, as it shows only a silhouette of the arterial lumen. Thus we cannot exclude 
the possibility that some of the perfusion abnormalities in patients without angiographically significant coronary artery disease represent true ischaemia. A study of coronary flow reserve in these patients could perhaps have provided more insight into the significance of such abnormalities.

\section{CONCLUSIONS}

Exercise SPECT myocardial perfusion imaging using $99 \mathrm{~m}$ technetium labelled agents is an accurate method for diagnosing and localising coronary artery disease in hypertensive patients. The diagnostic accuracy of exercise SPECT in hypertensive patients is comparable with that in normotensive patients. These data indicate that in the usual clinical setting, the accuracy of exercise myocardial perfusion scintigraphy is not reduced in hypertensive patients.

1 Gibbons RJ, Balady GJ, Beasley JW, et al. ACC/AHA guidelines for exercise testing. A report of the American College of Cardiology/American Heart Association task force on practice guidelines (committee on exercise testing). $7 \mathrm{Am}$ Coll Cardiol 1997;30:260-311.

2 Maddahi J, Kiat $\mathrm{H}$, Van Train KF, et al. Myocardial perfusion imaging with technetium-99m sestamibi SPECT in the evaluation of coronary artery disease [review]. Am $\mathcal{F}$ Cardiol 1990;66:55E-62E.

3 Berman DS, Kiat H, Van Train K, et al. Technetium $99 \mathrm{~m}$ sestamibi in the assessment of chronic coronary artery disease. Semin Nucl Med 1991;21:190-212.

4 Joint National Committee. The sixth report of the Joint National Committee on prevention, detection, evaluation,
and treatment of high blood pressure. Arch Intern Med and treatment of high

5 Pringle SD, Dunn FG, Lorimer AR, et al. The role of nuclear cardiology in hypertension. Nucl Med Commun 1994;15:4-8.

6 Houghton JL, Frank MJ, Carr AA, et al. Relations among impaired coronary flow reserve, left ventricular hypertrophy and thallium perfusion defects in hypertensive patients without obstructive coronary artery disease. $7 \mathrm{Am}$ Coll Cardiol 1990;15:43-51

7 Aguirre JM, Rodriguez E, Ruiz De Azua E, et al. Segmentary coronary reserve in hypertensive patients with echocardiographic left ventricular hypertrophy, gammagraphic ischemia and normal coronary angiography. Eur Heart f 1993;14(suppl J):25-31.

8 DePuey EG, Guertler-Krawczynska E, Perkins JV, et al. Alteration in myocardial thallium-201 distribution in Alteration in myocardial thallium-201 distribution in single-photon emission computed tomography. Am Cardiol 1988;62:234-8.

9 Frohlich ED, Apstein C, Chobanian AV, et al. The heart in hypertension. N Engl f Med 1992;327:998-1008.

10 Harrison DG, Marcus ML, Dellsperger KC, et al. Pathophysiology of myocardial perfusion in hypertension. Circulation 1991;83(suppl 5):III14-18.

11 Hamouda MS, Kassem HK, Salama M, et al. Evaluation of coronary flow reserve in hypertensive patients by dipyridamole transesophageal Doppler echocardiography. Am $f$ Cardiol 2000;86:305-8.

12 Baptista J, Arnese M, Roelandt JRTC, et al. Quantitative coronary angiography in the estimation of the functional
significance of coronary stenosis: Correlation with dobutamine-atropine stress test. 7 Am Coll Cardiol 1994;23: 1434-9.

13 Troy BL, Pombo J, Rackley CE. Measurement of left ventricular thickness and mass by echocardiography. Circuventricular thickness and

14 Devereux RB, Alonso DR, Lutas EM, et al. Echocardiographic assessment of left ventricular hypertrophy: comparison to necropsy findings. Am f Cardiol 1986;57:450-8

15 Levy D, Savage DD, Garrison RJ, et al. Echocardiographic criteria of left ventricular hypertrophy: the Framingham Heart Study. Am f Cardiol 1987;59:956-60.
16 Tarazi RC, Fouad FM. Assessment of cardiac status in hypertensive patients. $\mathcal{F}$ Hypertens 1985;3:S27-31.

17 Osbakken MD, Okada RD, Boucher CA, et al. Comparison of exercise perfusion and ventricular function imaging: an analysis of factors affecting the diagnostic accuracy of each technique. $\mathcal{F}$ Am Coll Cardiol 1984;3:272-83.

18 Cecil MP, Pilcher WC, Eisner RL, et al. Absence of defects in SPECT thallium 201 myocardial images in patients with systemic hypertension and left ventricular hypertrophy. $\mathrm{Am}$ f Cardiol 1994;74:43-64.

19 Schulman DS, Francis CK, Black HR, et al. Thallium 201 stress imaging in hypertensive patients. Hypertension 1987; 10:16-21.

20 Grogan M, Christian TF, Miller TD, et al. The effect of systemic hypertension on exercise tomographic thallium-201 imaging in the absence of electrocardiographic left ventricular hypertrophy. Am Heart 7 1993;126:327-32.

21 Chin WL, O'Kelly B, Tubau JF, et al. Diagnostic accuracy of exercise thallium-201 scintigraphy in men with asymptomatic essential hypertension. Am f Hypertens 1992;5:46572

22 Fragasso G, Lu C, Dabrowski P, et al. Comparison of stress/ rest myocardial perfusion tomography, dipyridamole and dobutamine stress echocardiography for the detection of coronary disease in hypertensive patients with chest pain and positive exercise test. 7 Am Coll Cardiol 1999;34:441-7.

23 Zouridakis EG, Cox ID, Garcia-Moll X, et al. Negative stress echocardiographic responses in normotensive and hypertensive patients with angina pectoris, positive exercise stress testing and normal coronary arteriograms. Heart 2000;83:141-6.

24 Elhendy A, Geleijnse ML, van Domburg RT, et al. Comparison of dobutamine stress echocardiography and 99 m-technetium sestamibi SPET for the diagnosis of coronary artery disease in hypertensive patients with and without left ventricular hypertrophy. Eur $\mathcal{F}$ Nucl Med 1998; 25:69-78

25 Elhendy A, van Domburg RT, Bax JJ, et al. Myocardial perfusion abnormalities in treated hypertensive patients without known coronary artery disease. F Hypertens 1999;17: 1601-6.

26 Kao CH, Wang SJ, Ting CT, et al. Tc-99m sestamibi myocardial SPECT in syndrome X. Clin Nucl Med 1996;21: 280-3.

27 Agabiti-Rosei E, Muiesan ML, Rizzoni D. Cardiovascular structural alterations in hypertension: effect of treatment. Clin Exp Hypertens 1996;18:513-22.

28 Santana-Boado C, Candell-Riera J, Castell-Conesa J, et al. Diagnostic accuracy of technetium-99m-MIBI myocardial SPECT in women and men. 7 Nucl Med 1998;39:751-5.

29 Van Train KF, Garcia EV, Maddahi J, et al. Multicenter trial validation for quantitative analysis of same-day rest-stress technetium-99m-sestamibi myocardial tomograms. $\mathcal{F} \mathrm{Nucl}$ Med 1994;35:609-18.

30 Rubello D, Zanco P, Candelpergher G, et al. Usefulness of $99 \mathrm{mTc}$-MIBI stress myocardial SPECT bull's-eye quantification in coronary artery disease. Q F Nucl Med 1995;39: 111-15.

31 Solot G, Hermans J, Merlo P, et al. Correlation of 99Tcmsestamibi SPECT with coronary angiography in general hospital practice. Nucl Med Commun 1993;14:23-9.

32 Zaret BL, Rigo P, Wackers FJ, et al. Myocardial perfusion imaging with $99 \mathrm{mTc}$ tetrofosmin. Comparison to $201 \mathrm{Tl}$ imaging and coronary angiography in a phase III multicenter trial. Tetrofosmin international trial study group. Circulation 1995;91:313-19.

33 Azzarelli S, Galassi AR, Foti R, et al. Accuracy of $99 \mathrm{mTc}$-tetrofosmin myocardial tomography in the evaluation of coronary artery disease. $\mathrm{F} \mathrm{Nucl} \mathrm{Cardiol} \mathrm{1999;6:183-}$ 9.

34 Montz R, Perez-Castejon MJ, Jurado JA, et al. Technetium$99 \mathrm{~m}$ tetrofosmin rest/stress myocardial SPET with a sameday 2-hour protocol: comparison with coronary angiography. A Spanish-Portuguese multicentre clinical trial. Eur 7 Nucl Med 1996;23:639-47.

35 Acampa W, Cuocolo A, Sullo P, et al. Direct comparison of technetium 99m-sestamibi and technetium 99mtetrofosmin cardiac single photon emission computed tomography in patients with coronary artery disease. $\mathcal{F ~ N u c l}$ Cardiol 1998;5:265-74

36 Flamen P, Bossuyt A, Franken PR. Technetium-99mtetrofosmin in dipyridamole-stress myocardial SPECT imaging: intraindividual comparison with technetium99m-sestamibi. F Nucl Med 1995;36:2009-15. 